\title{
Non-Coherent Direction of Arrival Estimation from Magnitude-Only Measurements
}

\author{
Haley Kim, Student Member, IEEE, Alexander M. Haimovich, Fellow, IEEE, and Yonina C. Eldar, Fellow, IEEE
}

\begin{abstract}
We consider the classical Direction of arrival (DOA) estimation problem in the presence of random sensor phase errors are present at each sensor. To eliminate the effect of these phase errors, we propose a DOA recovery technique that relies only on magnitude measurements. This approach is inspired by phase retrieval for applications in other fields. Ambiguities typically associated with phase retrieval methods are resolved by introducing reference targets with known DOA. The DOA estimation problem is formulated as a nonlinear optimization in a sparse framework, and is solved by the recently proposed GESPAR algorithm modified to accommodate multiple snapshots. Numerical results demonstrate good DOA estimation performance. For example, the probability of error in locating a single target within 2 degrees is less than 0.1 for $S N R \geq 15 \mathrm{~dB}$ and one snapshot, and negligible for $\mathrm{SNR} \geq 10 \mathrm{~dB}$ and five snapshots.
\end{abstract}

Index Terms - Direction of arrival, phase retrieval, sparsity.

\section{INTRODUCTION}

A N ASSUMPTION often relied upon in the literature on DOA problems is that ideal phase synchronization is available at the elements of the array. In practice, however phase synchronization is difficult to achieve and greatly increases hardware complexity. There are many causes that may lead to imperfect phase synchronization, such as phase noise of the local oscillator [14], [15], errors in antenna element placing [16], [17], and errors in downsampling due to clock drifting by the local oscillator [18]. When one tries to apply well known DOA estimation methods, such as MUSIC [5] or ESPRIT [19], in the presence of phase errors, the methods behave as if there is a severe degradation in the signal-to-noise ratio (SNR) [10]. Furthermore, when the phase errors exceed approximately $1 / 10$ of a cycle, coherency is lost, and the DOA cannot be accurately estimated irrespective of the SNR.

Recently, the topic of phase retrieval has been receiving increased attention in signal processing literature [20]. Phase retrieval refers to the problem in which one seeks to recover a

Manuscript received September 14, 2014; revised November 14, 2014; accepted November 21, 2014. Date of publication December 04, 2014; date of current version December 11, 2014. The associate editor coordinating the review of this manuscript and approving it for publication was Prof. Rodrigo C. de Lamare.

H. Kim and A. M. Haimovich are with New Jersey Institute of Technology, Newark, NJ 07102 USA (e-mail: hk83@njit.edu; haimovic@njit.edu).

Y. C. Eldar is with the Technion-Israel Institute of Technology, Haifa 32000, Israel (e-mail: yonina@ee.technion.ac.il).

Color versions of one or more of the figures in this paper are available online at http://ieeexplore.ieee.org.

Digital Object Identifier 10.1109/LSP.2014.2377556 (complex) signal from magnitude only measurements so that all phase information is lost. This problem originated in fields such as optical imaging [1], crystallography, [2] and others [3], where phase measurements are impossible to obtain. Solutions to the phase retrieval problem generally allow for various ambiguities considered acceptable in many applications, such as imaging and crystallography [4], [6], [7]. Applying a phase retrieval approach to the DOA estimation problem might free the system designer from the phase synchronization task, thus significantly reducing hardware complexity. Nevertheless, the ambiguities, usually associated with phase retrieval solutions would make the DOA estimation nonsensical.

In this letter, we propose a method for solving the DOA problem without phase information by relying on phase retrieval techniques. We refer to our method as non-coherent $D O A$ estimation, referring to an array with sensors that are not phase synchronized. We further introduce means to rid the estimates of ambiguities. We show, that at a small cost to performance, we can perform DOA estimation that is unaffected by phase synchronization errors.

The letter is organized as follows: the signal model in Section II, non-coherent DOA estimation in Section III, numerical examples are presented in Section IV, and concluding remarks are found in Section V.

Throughout, bold lowercase letters denote column vectors, and bold uppercase letters denote matrices; $\|\cdot\|_{F}^{2}$ denotes the Frobenius norm, while $\|\mathbf{X}\|_{0}$ counts the number of nonzero rows in $\mathbf{X}$.

\section{Signal Model}

Consider a linear antenna array of $N$ elements, where each element collects $P$ measurements of signals emanating from $K$ stationary targets in the far field. The array could be a passive array or part of a radar system. A set of measurements taken by the array sensors at a given time instant is known as a snapshot. The $n$-th array element is located at coordinate $z_{n}$ in an arbitrary coordinate system. The number of targets is assumed known, and the targets are assumed to be stationary. The signals in the system are of known carrier wavelength $\lambda$ and of negligible bandwidth, such that the pulse shape of the received signal does not contribute meaningful information to localization. For a target with DOA $\theta$, the signal received at the $n$-th element is given by $x e^{j(2 \pi / \lambda) z_{n} \sin \theta}$, where $x$ is the target's complex gain. For notational brevity, let $u=\sin \theta$ and $\psi=(2 \pi / \lambda) u$. With this notation, the response across the array from a unity gain target is given by

$$
\mathbf{a}(\psi)=\left[e^{j z_{1} \psi}, \ldots, e^{j z_{N} \psi}\right]^{T} .
$$


Then, a snapshot at time $t_{p}$ of the baseband response at the array due to the $K$ targets is given by the $N \times 1$ vector

$$
v\left(t_{p}\right)=\sum_{i=1}^{K} x_{i}\left(t_{p}\right) \mathbf{a}\left(\psi_{i}\right)
$$

where, $x_{i}\left(t_{p}\right)$ is the complex target gain for a target in position $\psi_{i}$ which can vary in time from snapshot to snapshot. Concatenating $P$ snapshots horizontally yields us the $N \times P$ measurement matrix $\Upsilon$ with the columns $v\left(t_{p}\right)$. We assume that the target's possible DOAs comply with a grid of $G$ points, with $G \gg K$, and define an $N \times G$ dictionary matrix A with columns $\mathbf{a}\left(\psi_{i}\right)$. The signal model is then given by,

$$
\mathbf{\Upsilon}=\mathbf{A X}+\mathbf{E}
$$

where $\mathbf{X}$ is sparse in the sense that it has only $K \ll G$ non-zero rows representing target gains, and the elements of $\mathbf{E}$ constitute spatio-temporal, complex-valued, additive white Gaussian noise (AWGN) with mean zero and variance $\sigma_{e}^{2}$. Our goal is to determine the $K$ non-zero rows indices, i.e. support, of $\mathbf{X}$, from the knowledge of the measurements $\mathbf{\Upsilon}$ and matrix $\mathbf{A}$. The support of $\mathbf{X}$ has a one-to-one relation with the $K$ DOA's $\psi_{k}$, $k=1, \ldots, K$. Sensor phase errors are modeled by a diagonal matrix $\mathbf{D}$ with elements $\left\{e^{j \phi_{n}}\right\}_{n=1}^{N}$, where $\phi_{n}$ is the phase error associated with sensor $n$. Thus the received signal model in the presence of phase errors is

$$
\mathbf{\Upsilon}=\mathbf{D A X}+\mathbf{E}
$$

\section{NON-COHERENT DiRECTION OF ARRIVAL ESTIMATION}

There is an extensive body of literature on solutions to the DOA problem. In general, these solutions assume phase synchronization at the array elements. It is well known that phase synchronization errors degrade DOA estimation performance. For example, it is shown in [8] that at high SNR, phase errors dominate the DOA estimation performance if the variance of the phase errors $\sigma_{\phi}^{2}>1 / \mathrm{SNR}$, where SNR is the effective signal to noise ratio (i.e. it incorporates the effect of using multiple observations). This means that for a $\mathrm{SNR}=20 \mathrm{~dB}$, phase errors larger than 6 degrees become important. In another example, it is shown in [9] that a 40-element array can easily distinguish between two sources separated by 2 degrees with ideal phase information, but the sources become indistinguishable for phase errors of 10 degrees. This motivates introducing an approach to DOA estimation that does not rely on signal phase, and hence is robust to phase synchronization errors.

Taking the magnitude squared of the measurements in (3), we obtain

$$
\mathbf{Y}=|\mathbf{D A X}+\mathbf{E}|^{2}
$$

where $\mathbf{Y}=|\mathbf{\Upsilon}|^{2}$, and the absolute value operator is applied element-wise to the matrix $\mathbf{\Upsilon}$. Recalling that $\mathbf{D}$ is a diagonal matrix of phase errors, in the noiseless case,

$$
|\mathbf{D A X}|^{2}=|\mathbf{A X}|^{2}
$$

which means that phase errors do not affect the measured signal. Therefore, we propose estimating the support of $\mathbf{X}$ directly from Y.

\section{A. Ambiguities}

To develop intuition for DOA estimation from magnitudeonly measurements, consider the case of two targets $i=1,2$ with bearings $\theta_{i}, \psi_{i}=(2 \pi / \lambda) \sin \theta_{i}$ and complex gains $x_{i}$. It is instructive to rewrite the complex gains $x_{i}=\left|x_{i}\right| e^{j \gamma_{i}}$ where $\gamma_{i}$ is the phase of the complex gain $x_{i}$. The noise-free signal received at the $n$-th sensor is given by

$$
\begin{aligned}
y_{n} & =|| x_{1}\left|e^{j\left(z_{n} \psi_{1}+\gamma_{1}\right)}+\right| x_{2}\left|e^{j\left(z_{n} \psi_{2}+\gamma_{2}\right)}\right|^{2} \\
& =\left|x_{1}\right|^{2}+\left|x_{2}\right|^{2}+2\left|x_{1}\right|\left|x_{2}\right| \cos \left(z_{n} \Delta \psi+\Delta \gamma\right)
\end{aligned}
$$

where $\Delta \psi=\psi_{2}-\psi_{1}$ and $\Delta \gamma=\gamma_{2}-\gamma_{1}$. To focus on the estimation of the DOA angles, assume $\left|x_{1}\right|$ and $\left|x_{2}\right|$ are known. Then $\Delta \gamma$ and $\Delta \psi$ may be estimated from equations (6) for $n=1, \ldots, N$. The estimate of $\Delta \psi$ is subject to several types of ambiguities. To simplify the discussion on ambiguities, let $\left|x_{1}\right|=\left|x_{2}\right|=1 / \sqrt{2}$. In this case, (6) becomes

$$
y_{n}=1+\cos \left(z_{n} \Delta \psi+\Delta \gamma\right) .
$$

The solution $\Delta \psi$ to (7) is subject to phase shift ambiguities, phase mirroring, and under some conditions, the response of the non-coherent array might contain grating lobes. A phase shift ambiguity occurs since for any phase shift $\delta_{\psi}, \Delta \psi=\psi_{1}-\psi_{2}=$ $\left(\psi_{1}+\delta_{\psi}\right)-\left(\psi_{2}+\delta_{\psi}\right)$. A phase mirroring ambiguity stems from the fact that if $\left(z_{n} \Delta \psi+\Delta \gamma\right)$ is a solution to the DOA estimation problem, then so is $-\left(z_{n} \Delta \psi+\Delta \gamma\right)$. The significance of the mirroring ambiguity is that given a reference target known DOA $\psi_{1}$, and an estimate of $\Delta \psi$ from solving (7), there are two possible $\psi_{2}$ DOA solutions for the unknown target, one to the left, and the other to the right of the reference target. The next lemma addresses the shift and mirroring ambiguities.

Lemma 1: Given one or more time-synchronized (but not necessarily phase-synchronized) sensors capable of performing signal magnitude measurements, the DOA of a single target may be estimated unambiguously if the DOA is known for at least a single reference target placed at either edge of the field of view of interest.

Proof: Given a known DOA $\psi_{r e f}$ for a reference target, set $\psi_{1}=\psi_{r e f}$, in (7). This forces $\delta_{\psi}=0$, resolving the shift ambiguity. Moving on to the mirroring ambiguity, both $\pm\left(z_{n}\left(\psi_{2}-\psi_{\text {ref }}\right)+\Delta \gamma\right)$ produce the same measurement $y_{n}$ in (7). The mirroring ambiguity is traced back to the even property of the cosine function. As a result of this ambiguity, given a reference target at DOA $\theta_{\text {ref }}$ (recall that $\psi=(2 \pi / \lambda) \sin \theta$ ), solutions for $\theta_{2}$ may occur on either side of $\theta_{\text {ref }}$. This ambiguity is resolved if targets are restricted to only one side of $\theta_{\text {ref }}$.

Note that consistent with our magnitude-only measurements assumption, the phase of $x_{r e f}$, say $\gamma_{r e f}$, cannot be measured and is unknown. In addition note that the estimation of $\Delta \psi$ is completely determined by the argument of the cosine term and the amplitude of $x_{r e f}$ does not play a role. This means the amplitude of $x_{r e f}$ does not affect the estimation of $\Delta \psi$. 


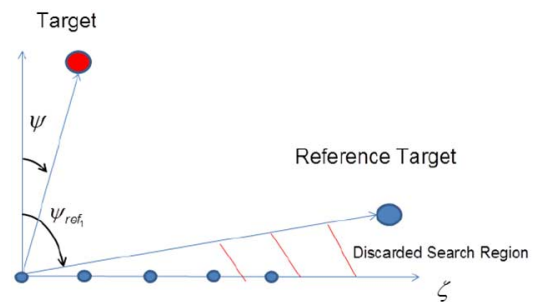

Fig. 1. Example use of a reference target to resolve mirroring ambiguities in non-coherent DOA estimation.

Fig. 1 illustrates how placing the reference target close to one end of the visible region $(-\pi / 2, \pi / 2)$, resolves the mirroring ambiguity, at the cost of a small loss in the visible region.

In the case of multiple targets with unknown DOA, an additional ambiguity arises when multiple pairs of targets yield the same $\Delta \psi$. Intuitively, the chances of this type of ambiguity may be reduced by using multiple reference targets.

\section{B. DOA Recovery}

Based on (4) we propose estimating the DOAs by considering the optimization problem,

$$
\min _{\mathbf{X}}\left\|\mathbf{Y}-|\mathbf{A X}|^{2}\right\|_{F}^{2} \quad \text { s.t. }\|\mathbf{X}\|_{0} \leq K+K_{r e f}, S_{r e f} \subseteq S
$$

where $K$ is the number of unknown targets, while $K_{\text {ref }}$ is the number of reference targets. As previously discussed, if one reference target is used, it must lie on one end of the area of interest. Points of the other side of this boundary, must be discarded. This is equivalent to removing columns of $\mathbf{A}$ that correspond to the grid points that must be discarded. This ensures that the solution is restricted to one side of the reference target.

The set $S_{r e f}$ is the set of indices that specifies the location(s) of the reference target(s) or equivalently the rows in $\mathrm{X}$ that correspond to the reference targets. The set $S$ is the set of indices of all the targets or nonzero rows of $\mathbf{X}$. The constraint $S_{\text {ref }} \subseteq S$ forces the estimated support $S$ to contain the reference targets. To solve (8), we modify GESPAR [7], a phase retrieval algorithm that exploits sparsity. GESPAR was designed for a single snapshot, but here we extend it to handle multiple snapshots and to use the DOA information of the reference targets. Before we discuss the improvements to GESPAR in more detail, we outline a short description of the algorithm (refer to [7] for more details).

GESPAR may be applied to solve (8) for a single snapshot, i.e. $\mathbf{Y}=\mathbf{y}$, where $\mathbf{y}$ is an $N \times 1$ measurement vector, and $\mathbf{X}=\mathbf{x}$, where $\mathbf{x}$ is a $G \times 1$ vector of target gains. The algorithm begins by randomly choosing $K+K_{\text {ref }}$ indices and places them into $S$. Once an initial guess of the support is made, the cost function becomes

$$
f\left(\mathbf{x}_{S}\right)=\min _{\mathbf{x}_{S}}\left\|\mathbf{y}-\left|\mathbf{A}_{S} \mathbf{x}_{S}\right|^{2}\right\|_{2}^{2}
$$

where $\mathbf{A}_{S}$ is formed from the columns of $\mathbf{A}$ that correspond to the support set $S$, and similarly $\mathbf{x}_{S}$ corresponds to the elements of $\mathbf{x}$ in the support $S$. The unconstrained optimization problem (9) is solved via the Damped Gauss-Newton (DGN) algorithm [13] to determine the elements of $\mathbf{x}_{S}$ and to evaluate the value of the cost function. The vector $\mathbf{x}$ is then created from the estimate $\mathbf{x}_{S}$, using the equation $\mathbf{x}=\mathbf{U}_{S} \mathbf{x}_{S}$, where $\mathbf{U}_{S} \in \mathbb{R}^{G \times\left(K+K_{r e f}\right)}$ is a matrix consisting of the columns of the identity matrix $\mathbf{I}_{G}$ corresponding to the set $S$. Note that (9) does not require the complex amplitude of the reference target(s) and we only require that the target gain is nonzero. A local search is then performed for a new support set that achieves a lower cost function value; for details see [7]. This process continues until a cost function value is achieved that is below a user defined threshold or a predetermined maximum number of swaps is exhausted.

Our modified version of GESPAR follows the same procedure as GESPAR, but accounts for multiple snapshots, which GESPAR cannot accommodate. Specifically:

- We find the coefficients of $\mathbf{X}_{S}$ column by column by solving $P$ optimization problems of the form $f\left(\mathbf{x}^{i}\right)=\min _{\mathbf{x}_{S}^{i}}\left\|\mathbf{y}^{i}-\left|\mathbf{A}_{S} \mathbf{x}_{S}^{i}\right|^{2}\right\|_{2}^{2}$ using the DGN method, where the superscript $i$ represents the $i$-th column of a matrix. The matrix $\mathbf{X}$ is generated from $\mathbf{X}=\mathbf{U}_{S} \mathbf{X}_{S}$. The cost function value $f\left(\mathbf{X}_{S}\right)$ is evaluated from, $f\left(\mathbf{X}_{S}\right)=\sum_{i=1}^{P} f\left(\mathbf{x}_{S}^{i}\right)$.

- To accommodate $P$ snapshots in the definition of the gradient (see step 1 above), we define $\mathbf{g}=\sum_{i=1}^{P}\left|\nabla f\left(\mathbf{x}^{i}\right)\right|$. Here $\nabla f\left(\mathbf{x}^{i}\right)$ is the gradient of the cost function $f\left(\mathbf{x}^{i}\right)=$ $\left\|\mathbf{y}^{i}-\left|\mathbf{A} \mathbf{x}^{i}\right|^{2}\right\|_{2}^{2}$ with respect to $\mathbf{x}^{i}$.

\section{NUMERICAL EXAMPLES}

In this section, numerical examples are presented to illustrate the performance of non-coherent DOA estimation. For reference, results are also shown for coherent DOA estimation under the assumption that the targets are sparse. The coherent case is solved by applying the orthogonal matching pursuit (OMP) algorithm [12]. Unless specified otherwise, the phase error matrix $\mathbf{D}$ is taken to be the identity matrix I corresponding to no phase errors. Common to Figs. $2-3$ is a linear array of $N=20$ elements spaced at half-wavelength of the carrier frequency. For these figures, a grid of $G=30$ points is uniformly spaced over the sector $[\sin 0, \sin \pi / 2]$. The localization problem is solved using observations from either $P=1$ or $P=5$ snapshots. For scenarios in which a single target $K=1$ is to be localized, a reference target of known DOA and amplitude is found at $\sin \theta_{\text {ref }}=0$. For problems where multiple targets are estimated, there are two known reference targets at respectively, $\sin \theta_{r e f_{1}}=0$ and $\sin \theta_{r e f_{2}}=0.035$ (corresponding to adjacent grid points) it was observed that this configuration resolved the ambiguities during simulations for two targets. All reference and unknown targets have unit amplitude and random phase $U \sim[0,2 \pi]$, both of which are fixed over the observation interval. Signals from the targets are measured in the presence of additive, white, complex Gaussian noise with zero mean and variance $\sigma_{e}^{2}$. The SNR per sensor and observation is then defined SNR $=-10 \log _{10} \sigma_{e}^{2}$. The figure of merit used to evaluate performance is the probability of error defined as the ratio of incorrectly recovered signal supports to the number of experiments. A DOA estimate is considered in error whenever it is associated with any grid point other than the correct one.

Fig. 2 shows the probability of error of the DOA estimation versus SNR for coherent and non-coherent DOA estimation of 


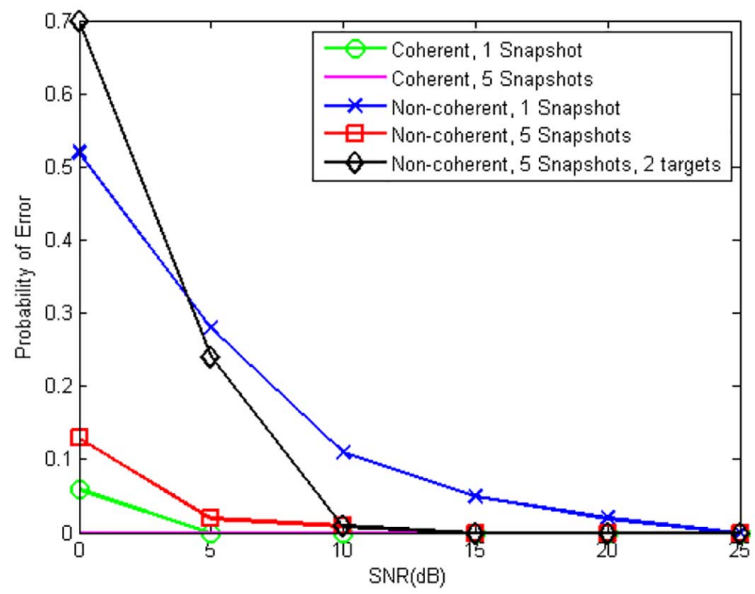

Fig. 2. Probability of error vs SNR for $N=20 \mathrm{~K}=1, G=30$. A reference target is placed at $\sin \theta_{r e f}=0$. No phase errors, $\mathbf{D}=\mathbf{I}$.

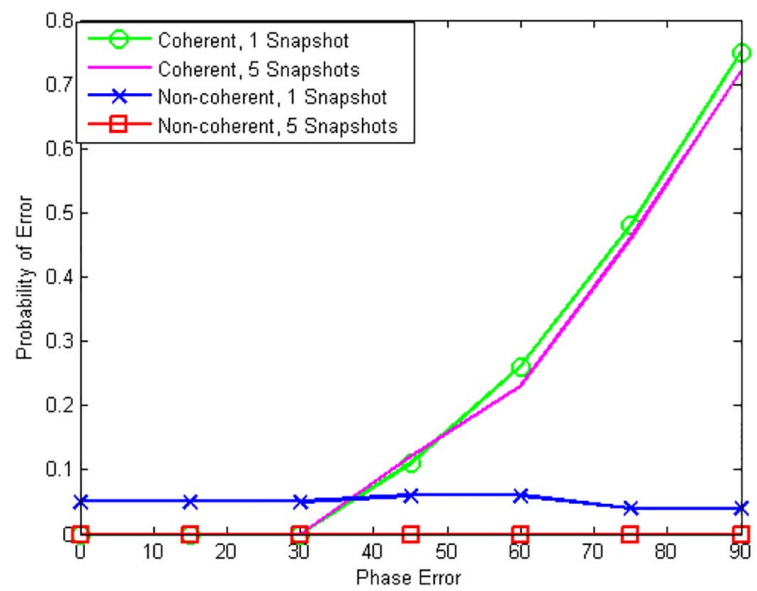

Fig. 3. Probability of error vs phase errors for $N=20, G=30, K=1$, $\mathrm{SNR}=15 \mathrm{~dB}$. A reference target is placed at $\sin \theta_{\text {ref }}=0$, and the unknown target is placed at $\sin \theta_{1}=0.035$. Both targets have amplitudes of one with random phase. The phase error $\phi$ is drawn from a Gaussian distribution that is independent from sensor to sensor.

one or two targets. In the single target case, the target is located at $\sin \theta=0.035$ one grid point away from the known reference target) in the two target case, targets are located at $\sin \theta_{1}=0.241$ and $\sin \theta_{2}=0.517$. The results are further parameterized by the number of observation snapshots. From the figure, it is observed that for $P=1$ snapshot, non-coherent DOA estimation has a probability of error lower than 0.1 for SNR $\geq 15 \mathrm{~dB}$. With $P=5$ snapshots, performance improves to an almost zero probability of error for $\mathrm{SNR} \geq 10 \mathrm{~dB}$. As expected, non-coherent DOA incurs a performance loss compared to DOA estimation that can exploit phase information.

Fig. 3 illustrates the effect of phase errors on coherent and non-coherent DOA estimation. The figure displays the probability of error for locating a single target as a function of the standard deviation of sensor phase errors, for $\mathrm{SNR}=15 \mathrm{~dB}$. The unknown target is located one grid point away from the reference target. Each entry of the phase error matrix $\mathbf{D}$ is equal to $e^{j \phi_{n}}$ where $\phi_{n}$ is drawn from a Gaussian distribution. This

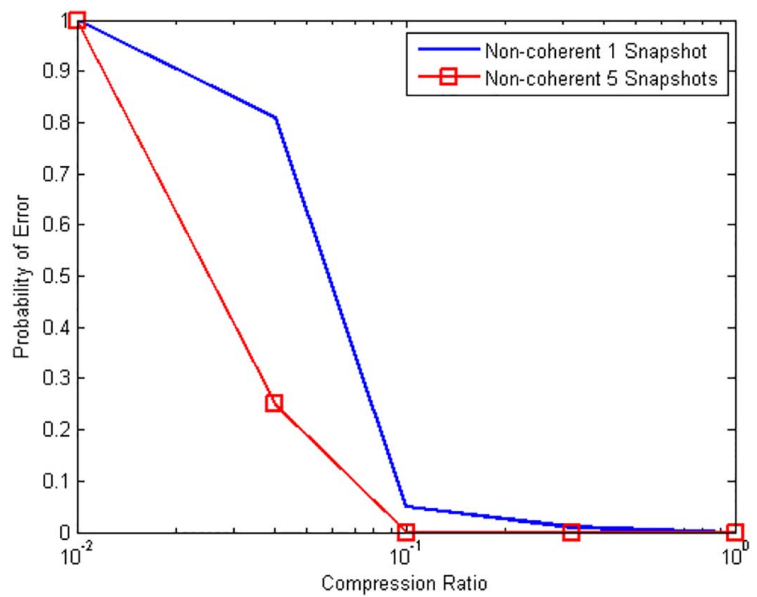

Fig. 4. Probability of error vs compression ratio for $G=100, Z=50 \lambda$. Sensor locations are drawn from a uniform distribution. A reference target is placed at $\sin \theta_{\text {ref }}=0$ and the unknown target is placed at an adjacent grid point, $\sin \theta_{1}=0.0101$. Both targets have amplitudes of one with random phase.

figure demonstrates a key benefit of non-coherent DOA estimation. It is observed that phase errors have no effect on non-coherent DOA, while the coherent method starts to collapse for phase errors larger than roughly $1 / 10$ of a cycle.

While the previous examples were generated with a filled array ( $\lambda / 2$ element spacing), in Fig. 4 the number of elements varies. The horizontal axis represents the compression ratio defined as the ratio of the number of elements in the array to the number of elements in a filled array. Here, the number of elements in the filled array was taken to be 100 . The elements are distributed along the array according to a uniform random variable $U \sim[0, Z]$, where $Z$ is the aperture size in wavelengths. The number of grid points is $G=100$. By placing the unknown target one grid point away from the reference target, the two targets may be resolved if the DOA performance reaches the resolution limit. The goal in this example is then to study whether the resolution limit may be reached with a number of elements significantly lower than that of a full array. From the figure, it is observed that probabilities of error lower than 0.05 are obtained for both single and five snapshots for a sparse array with as few as $1 / 10$ of the number of elements of a filled array. This figure demonstrates the ability of non-coherent, sparse arrays in conjunction with a sparse framework to support high resolution DOA estimation.

\section{Conclusions}

In this letter we proposed a new approach to solving the DOA problem that is insensitive to sensor phase error. The method uses a modified version of the GESPAR algorithm. We demonstrated through numerical simulations that accurate DOA estimation using a non-coherent method is possible with only a small penalty in performance. The performance penalty diminishes as SNR increases. We also showed that when phase errors are present in the system, coherent methods experience a degradation in performance as the variance of the phase errors increases. In contrast, because our non-coherent approach does not make use of phase information, it does not experience any degradation in performance irrespective of the phase errors. 


\section{REFERENCES}

[1] A. Walther, "The question of phase retrieval in optics," J. Mod. Opt., vol. 10 , no. 1, pp. 41-49, 1963.

[2] R. Harrison, "Phase problem in crystallography," J. Opt. Soc. Amer. A, vol. 10, no. 5, pp. 1046-1055, 1993.

[3] N. Hurt, P. Retrieval, and Z. Crossings, Phase Retrieval and Zero Crossings: Mathematical Methods in Image Reconstruction. Berlin, Germany: Springer-Verlag, 2001, vol. 52.

[4] J. Fienup, "Phase retrieval algorithms: A comparison," Appl. Opt., vol. 21, no. 15 , pp. 2758-2769, 1982.

[5] R. O. Schmidt, "Multiple emitter location and signal parameter estimation," IEEE Trans. Antennas Propagat., vol. 34, no. 3, pp. 276-280, Mar. 1986.

[6] E. Candes, Y. C. Eldar, T. Strohmer, and V. Voroninski, "Phase retrieval via matrix completion," SIAM J. Imag. Sci., vol. 6, no. 1, pp. 199-225, Feb. 2013.

[7] Y. Shechtman, A. Beck, and Y. C. Eldar, "GESPAR: Efficient phase retrieval of sparse signals," IEEE Trans. Signal Process., vol. 62, no. 4, pp. 928-938, Feb. 15, 2014.

[8] A. L. Swindlehurst and T. Kailatxh, "A performance analysis of subspace-based methods in the presence of model errors. I. The MUSIC algorithm," IEEE Trans. Signal Process., vol. 40, no. 7, pp. 1758-1774, Jul. 1992.

[9] M. P. Wylie, S. Roy, and H. Messer, "Joint DOA estimation and phase calibration of linear equispaced (LES) arrays," IEEE Trans. Signal Process., vol. 42, no. 12, pp. 3449-3459, Dec. 1994.

[10] L. Godara, "The effect of phase-shifter errors on the performance of an antenna-array beamformer," IEEE J. Oceanic Eng., vol. 10, no. 3, pp. 278-284, Jul. 1985.
[11] J. H. Seldin and J. R. Fienup, "Numerical investigation of the uniqueness of phase retrieval," J. Opt. Soc. Amer. A, vol. 7, no. 3, pp. 412-427, 1990.

[12] J. A. Tropp and A. C. Gilbert, "Signal recovery from random measurements via orthogonal matching pursuit," IEEE Trans. Inf. Theory, vol. 53, no. 12, pp. 4655-4666, Dec. 2007.

[13] A. Bjorck, Numerical Methods for Least Squares Problems. Philadelphia, PA, USA: SIAM, 1996, p. 51

[14] G. Krieger and M. Younis, "Impact of oscillator noise in bistatic and multistatic SAR," IEEE Geoscience Remote Sens. Lett., vol. 3, no. 3, pp. 424-428, Jul. 2006.

[15] G. Krieger and A. Moreira, "Spaceborne bi- and multistatic SAR: Potential and challenges," Proc. Inst. Elect. Eng., Radar, Sonar, Navig., vol. 153, no. 3, pp. 184-198, Jun. 2006.

[16] A. Goffer and G. Langholz, "The performance of adaptive array in a dynamic environment," IEEE Trans. Aerosp. Electron. Syst., vol. AES-23, pp. 485-492, 1987.

[17] L. C. Godara, "Application of antenna arrays to mobile communications. II. Beam-forming and direction-of-arrival considerations," Proc. IEEE, vol. 85, pp. 1195-1245, Aug. 1997.

[18] M. Eineder, "Oscillator clock drift compensation in bistatic interferometric SAR," in Proc. IGARSS, Toulouse, France, 2003, pp. 1449-1451.

[19] R. Richard and T. Kailath, "ESPRIT-estimation of signal parameters via rotational invariance techniques," IEEE Trans. Acoust., Speech, Signal Process., vol. 37, pp. 984-995, 1989.

[20] Y. Shechtman, Y. C. Eldar, O. Cohen, H. N. Chapman, J. Miao, and M. Segev, "Phase retrieval with application to optical imaging," IEEE Signal Process. Mag., to be published. 